\title{
MINIATURE ROBUST HIGH-BANDWIDTH FORCE SENSOR WITH MECHANICALLY AMPLIFIED PIEZORESISTIVE READOUT
}

Dennis Alveringh ${ }^{1,2}$, Diana L. van der Ven ${ }^{1}$, Henk-Willem Veltkamp ${ }^{l}$, Kevin M. Batenburg ${ }^{1}$, Remco G. P. Sanders ${ }^{l}$, David Fernandez Rivas ${ }^{l}$, and Remco J. Wiegerink ${ }^{l}$

${ }^{1}$ MESA+ Institute, University of Twente, Enschede, THE NETHERLANDS and

${ }^{2}$ Salland Engineering B.V., Zwolle, THE NETHERLANDS

\begin{abstract}
For fast and accurate force measurements, e.g., sampling the impact of a liquid droplet, a highly sensitive high-bandwidth force sensor is required. High-sensitivity and high-bandwidth are contradictive specifications in recently published force sensors. The miniature force sensor proposed in this paper uses a novel combination of inplane sensing, mechanical amplification, and piezoresistive readout that overcomes this contradiction. The straightforward fabrication process consists of three photomasks and uses a single SOI wafer. The sensor has a proven range of $13 \mathrm{mN}$, a sensitivity of $1.46 \mathrm{~N}^{-1}$ and a bandwidth of $75 \mathrm{kHz}$. This is the first force sensor in this force range with such a large bandwidth and has the potential to open up a new field of force sensing where high sampling rates and low ranges are crucial.
\end{abstract}

\section{KEYWORDS}

Force sensor, load cell, microdroplet, needle-free injector, liquid jets, mechanical amplification.

\section{INTRODUCTION}

Understanding the microscale and microsecond impact behavior of liquids is crucial for various applications. Microdroplet impact plays a large role in, e.g., rain erosion of wind turbine blades, and within the inkjet printing field $[1,2]$. Furthermore, the use of liquid jet injections as alternative to needles is widely explored. A large variety of needle-free injectors exists, some of them are already commercialized while others are still in development [3]. To understand and model the injection behavior accurate impact-force measurements are required. The impact of larger volume devices can be measured using commercial load cells, due to longer impact times and higher associated impact forces $[4,5]$. Consequently, these devices typically result in deep (subcutaneous or intramuscular) injections and often cause pain and bruising. To prevent this, other devices use low volume jets and aim at superficial skin layers (epidermis or dermis) [6, 7]. Furthermore, dermal or epidermal injections have therapeutic benefits. For example, vaccine and insulin delivery within the epidermis and dermis, respectively, is reported to be more efficient compared to traditional delivery. [8, 9].

Recent studies from one of these systems rely on low volume microjets $(20 \mathrm{~nL}, 50 \mu \mathrm{m}$ diameter $)$, with estimated forces of $0.5 \mathrm{mN}$ to $5 \mathrm{mN}$ (depending on jetting velocity, typically $20 \mathrm{~m} \mathrm{~s}^{-1}$ to $70 \mathrm{~m} \mathrm{~s}^{-1}$ ) resulting in impact times between $100 \mu$ s to $300 \mu$ s $[7,10]$.

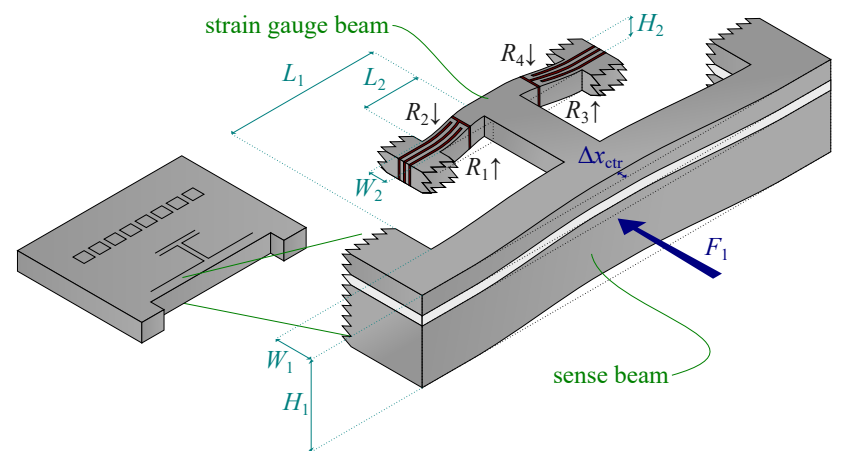

Figure 1: Illustration of the operation principle of the force sensor. A force applied on the clamped-clamped sense beam causes a mechanically amplified increase of strain in the strain gauge beam.

This class of microjets falls either below the sensitivity or temporal resolution of commercial devices, and thus impact forces can only be estimated using high speed recordings. Consequently, the actual force distribution over time cannot be characterized. Published micromachined load cells show high resolutions ( $\mathrm{nN}[11]$ to $\mu \mathrm{N}[12,13])$, but limited bandwidth $(<10 \mathrm{kHz})$. This paper describes a force sensing structure with piezoresistive readout and mechanical amplification that bridges this gap in measurement range.

\section{THEORY AND DESIGN}

The sensor consists of two clamped-clamped beams that are rigidly connected in the center as illustrated in Figure 1. The force is applied in-plane to the center of the sense beam. This force causes a displacement, which is transferred to the strain gauge beam. This beam consists of piezoresistive strain gauges in a Wheatstone bridge configuration. The strain in the strain gauge beam is higher compared to the strain in the sense beam as it is shorter. However, the latter beam does not significantly influence the stiffness or frequency behavior due to its lower width and height.

The second moment of area $I_{i}$ for the bending of both beams is equal to [14]:

$$
I_{i}=\frac{H_{i} W_{i}^{3}}{12},
$$

with $i=1$ for the sense beam and $i=2$ for the strain gauge beam, $H_{i}$ the height of the beam, and $W_{i}$ the width of the beam. The displacement in the center $\Delta x_{\text {ctr }}$ due to deformation caused by a force $F_{i}$ in the center of a clampedclamped beam can be derived using Euler-Bernoulli beam 
theory [14]:

$$
\Delta x_{\mathrm{ctr}}=\frac{F_{i} L_{i}^{3}}{192 E I_{i}}=\frac{F_{i} L_{i}^{3}}{16 E H_{i} W_{i}^{3}},
$$

with $L_{i}$ the length of the beam and $E$ Young's modulus of silicon in [110] direction. Note that $x_{\mathrm{ctr}}$ is equal for both beams as these are rigidly connected in the center. The stiffness $k_{i}$ can be approximated using Hooke's law by:

$$
k_{i}=\frac{F_{i}}{\Delta x_{\mathrm{ctr}}}=\frac{16 E H_{i} W_{i}^{3}}{L_{i}^{3}},
$$

The stiffness should be dominated by the sense beam, i.e., when $k_{1} \gg k_{2} \rightarrow k \approx k_{1}$. This gives the following relation for the dimensions of the beam:

$$
\frac{H_{1} W_{1}^{3}}{L_{1}^{3}} \gg \frac{H_{2} W_{2}^{3}}{L_{2}^{3}}
$$

The maximum strain $\varepsilon_{\max , i}$ for clamped-clamped beams with an applied force in the center is:

$$
\varepsilon_{\max , i}=\frac{\sigma_{\max , i}}{E}=\frac{F_{i} L_{i} W_{i} / 2}{8 E I_{i}}=\frac{3 F_{i} L_{i}}{4 E W_{i}^{2} H_{i}},
$$

with $\sigma_{\max , i}$ the maximum stress. Substitution of (2) into (5) gives:

$$
\varepsilon_{\max , i}=\frac{12 W_{i} \Delta x_{\mathrm{ctr}}}{L_{i}^{2}},
$$

which forms a straight-forward approximation for the stress in the strain gauges $\varepsilon_{\max , 2}$. The mechanical amplification can be derived from the maximum strain in the sense beam $\varepsilon_{\text {max }, 1}$ and the maximum strain in the strain gauge beam $\varepsilon_{\max , 2}$ :

$$
\frac{\varepsilon_{\max , 2}}{\varepsilon_{\max , 1}}=\frac{W_{2} L_{1}^{2}}{W_{1} L_{2}^{2}} \approx 5.6 .
$$

The bridge voltage of the Wheatstone bridge $V_{\mathrm{b}}$ is directly related to the relative change in resistance and is proportional to the maximum strain $\varepsilon_{\max , 2}$ :

$$
\frac{V_{\mathrm{b}}}{V_{\mathrm{s}}}=\left(\frac{R_{2}}{R_{1}+R_{2}}-\frac{R_{3}}{R_{3}+R_{4}}\right)=\frac{\Delta R}{R}=G \varepsilon_{\max , 2},
$$

with $R_{j}$ the resistors as indicated in Figure 1, $\Delta R$ the change in resistance due to the strain, $R$ the resistance in rest, and $G$ the gauge factor. The latter is dependent on the doping level and crystal orientation, but lies in the order of 10 to 100 [15].

A measure for the bandwidth of the sensor can be found by the resonance frequency $f_{0}$ of the first mode, which can be estimated by [16]:

$$
f_{0}=6.48 \frac{W_{1}}{2 \pi L_{1}^{2}} \sqrt{\frac{E}{\rho}}
$$

with $\rho$ the density of silicon.

The dimensions of the sensor components and material properties are given in Table 1. Filling in the numbers in (3), (8), and (9) gives a spring constant of $1.80 \cdot 10^{4} \mathrm{~N} \mathrm{~m}^{-1}$, a sensitivity of $1.39 \mathrm{~N}^{-1}$ and a resonance frequency of $110 \mathrm{kHz}$.

Simulations based on finite element method (FEM) were conducted using COMSOL Multiphysics ${ }^{\circledR}$ 5.6. The model consisted of both beams and transmission. It was based on the layer stack as described in the next section consisting of silicon dioxide $\left(\mathrm{SiO}_{2}\right)$ and crystalline silicon (anisotropic elasticity). The results correspond well to the analytical model with a spring constant of $1.87 \cdot 10^{4} \mathrm{~N} \mathrm{~m}^{-1}$, a sensitivity of $1.34 \mathrm{~N}^{-1}$, and a resonance frequency of $103 \mathrm{kHz}$.

Table 1: Dimensions of the sensor design as indicated in Figure 1 and material properties.

\begin{tabular}{lllll}
\hline Length & $L_{1}$ & $2 \mathrm{~mm}$ & $L_{2}$ & $0.6 \mathrm{~mm}$ \\
Width & $W_{1}$ & $50 \mu \mathrm{m}$ & $W_{2}$ & $25 \mu \mathrm{m}$ \\
Height & $H_{1}$ & $427 \mu \mathrm{m}$ & $H_{2}$ & $25 \mu \mathrm{m}$ \\
Young's modulus & $E$ & $169 \mathrm{GPa}$ & & \\
Density of silicon & $\rho$ & $2330 \mathrm{~kg} \mathrm{~m}^{-3}$ & & \\
Gauge factor & $G$ & 30 & & \\
\hline
\end{tabular}

\section{FABRICATION}

The fabrication is based on [17] with an additional step to realize piezoresistive strain gauges in the device layer. The device is fabricated in an SOI wafer with a highly p-type (boron) doped silicon handle layer of $400 \mu \mathrm{m}$, buried oxide layer of $2 \mu \mathrm{m}$, and a highly p-type doped silicon device layer of $25 \mu \mathrm{m}$ (Figure $2 \mathrm{a}$ ). The sense beam is realized in all three layers. The transmission and strain gauge beam are realized in the device layer only.

(a)

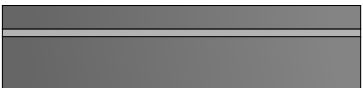

(h)

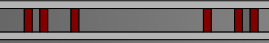

(b)

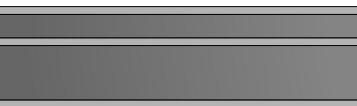

(i)

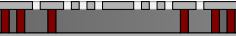

(c)
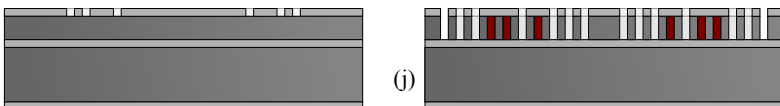

(d)

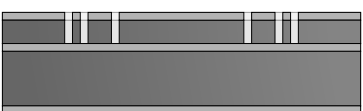

(j)

(e)

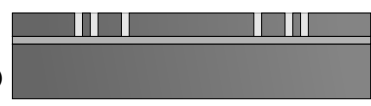

(k)

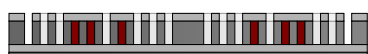

(f)

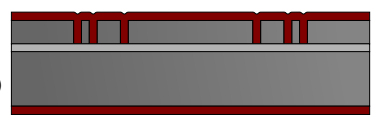

(m)

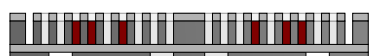

(1)

(g)

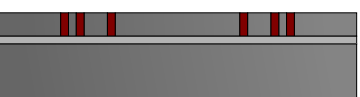

(n)
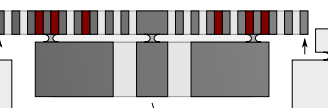

p-type silicon

$\square$ silicon dioxide

silicon rich nitride

Figure 2: Illustrations of the fabrication steps. The device has been fabricated by three high aspect ratio plasma etches, conformal silicon rich silicon nitride deposition, and one isotropic release etch.

A $\mathrm{SiO}_{2}$ layer of $1 \mu \mathrm{m}$ is deposited by wet thermal oxidation (Figure 2b). Directional reactive ion etching (RIE) is used to form the isolation mask in the $\mathrm{SiO}_{2}$ at the device layer side (Figure $2 \mathrm{c}$ ). Then, high-aspect ratio ( $3 \mu \mathrm{m}$ wide, $25 \mu \mathrm{m}$ deep) trenches with a slightly positive taper are 
realized using deep reactive ion etching (DRIE, Figure 2d). The hard mask is stripped using directional RIE on both sides of the wafer (Figure 2e). The trenches are filled with low-stress silicon rich silicon nitride (SiRN) using lowpressure chemical vapor deposition (LPCVD, Figure 2f). The SiRN on the surface is stripped using a directional RIE on both sides of the wafer (Figure $2 \mathrm{~g}$ ). As hard mask for the rest of the structures, a layer of $1 \mu \mathrm{m} \mathrm{SiO}$ is deposited using LPCVD and annealed (Figure 2h). Directional RIE is used to form the mask in the $\mathrm{SiO}_{2}$ layer on the device layer (Figure 2i). Then DRIE is used to form the structures in the device layer (Figure 2j). Similar RIE and DRIE steps are performed on the handle layer to form the hard mask and structures in the handle layer (Figures $2 \mathrm{k}$ and 21). The structures are released using an isotropic vapour-phase hydrogen fluoride (HF) etch (Figure $2 \mathrm{~m}$ ). This etch also released the dies from the wafer.

All photolithography steps have been performed with positive photoresist. Resist stripping has been performed by oxygen plasma. The chips are assembled using adhesive bonding on a printed circuit board (PCB) and wirebonded with aluminum wire (Figure 2n).

A scanning electron microscopy (SEM) image and a photograph of the sensor are shown in Figures 3 and 4 respectively.

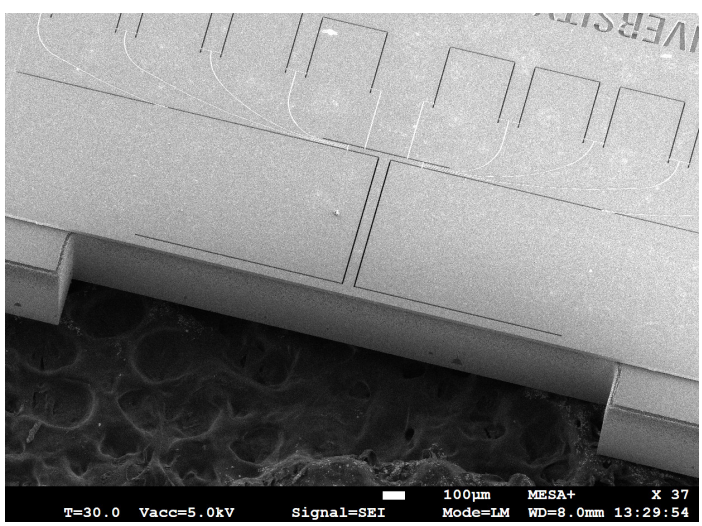

Figure 3: Scanning electron microscopy image of the device.

\section{CHARACTERIZATION}

The static performance of the sensor has been measured by applying a force range in increasing and decreasing order using a piezo actuator. The setup is illustrated in Figure 5. A lock-in amplifier (Stanford Research Systems ${ }^{\circledR}$ SR860) is used to apply the supply voltage ( $100 \mathrm{mV}$ amplitude) and to

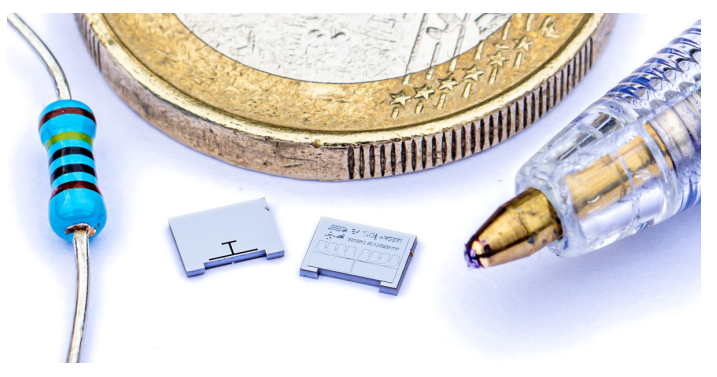

Figure 4: Photograph of the top and bottom of the fabricated force sensor. The chip measures $4 \mathrm{~mm}$ by $3 \mathrm{~mm}$.

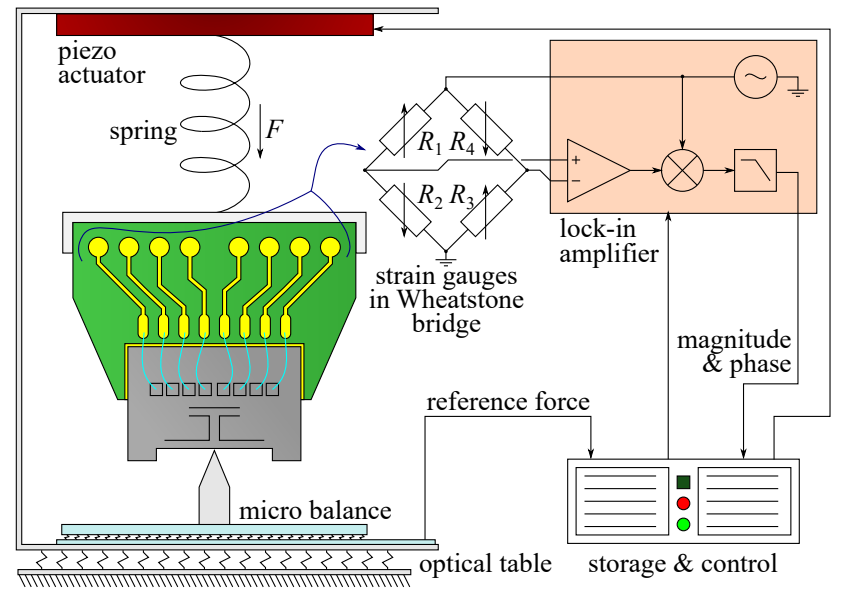

Figure 5: Schematic diagram of the measurement setup for the static characterization. A piezo actuator and spring apply force on the device under test and a microbalance reference. Lock-in amplification is used to detect the bridge voltage of the strain gauges.

measure the bridge voltage. For reference, a microbalance (Mettler Toledo® AT200) has been used.

The acquired results with a model fit based on equation (8) are shown in Figure 6. The total measurement time was approximately 3.5 hours. The results show that the sensor has a range of $13 \mathrm{mN}$ with high linearity $\left(R^{2}>0.9999\right)$, a sensitivity of $1.46 \mathrm{~N}^{-1}$ and a standard deviation-based accuracy of $100 \mu \mathrm{N}$.

The frequency behavior of the sensor has been measured by laser Doppler vibrometry (Polytec $®$ MSA-600) with external piezo actuation and is compared to the calculated resonance frequency and FEM simulations. Figure 7 shows that the resonance frequency for the first vibrational mode is at $75 \mathrm{kHz}$ which gives a measure for the bandwidth. Figure 8 shows the first three measured vibrational modes. The measured resonance frequency is lower than expected, which could be caused by non-ideal etching profiles.

A comparison of the measurement results with calculations and simulations is shown in Table 2.

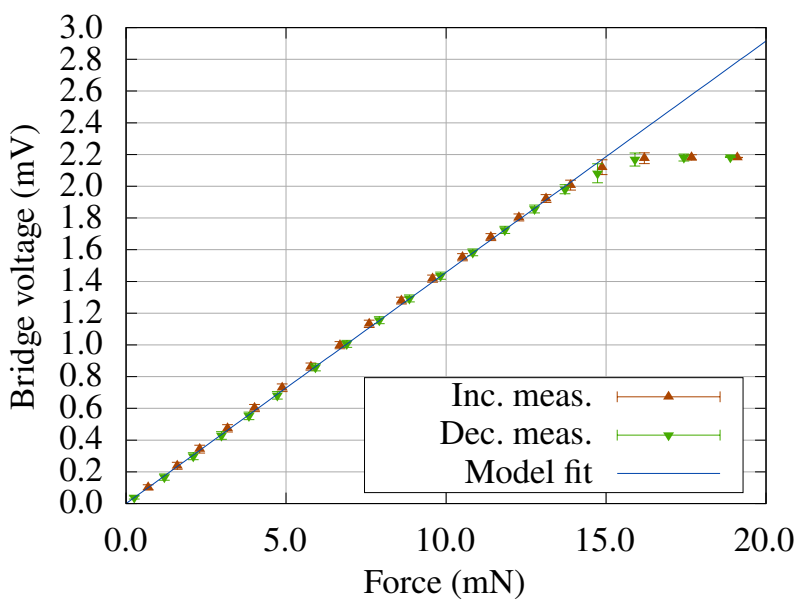

Figure 6: Measured bridge voltages (offset corrected) for applied forces with standard deviation (errorbars), in increasing $(\mathbf{\Delta})$ and decreasing $(\mathbf{\nabla})$ order compared with model fit (-). 


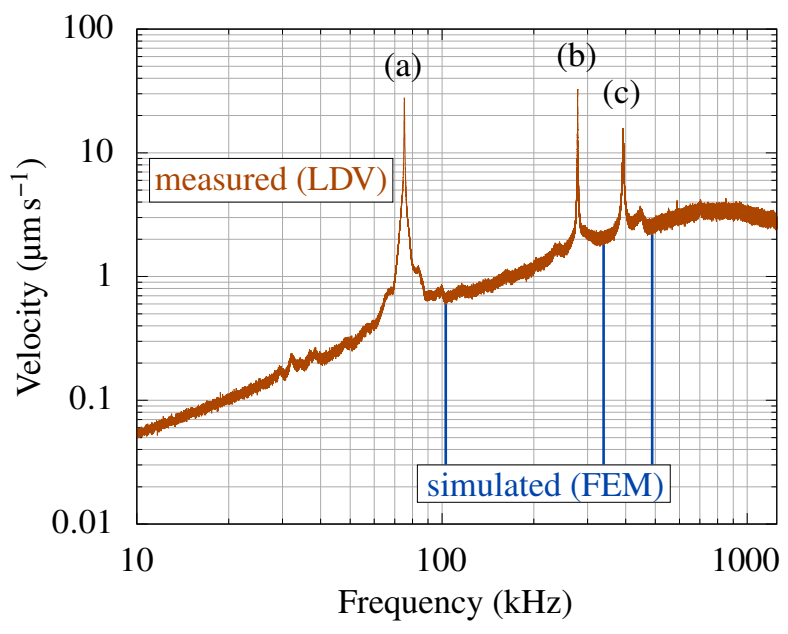

Figure 7: Frequency spectrum of averaged velocity magnitudes for all points on the sense beam measured using laser Doppler vibrometry and compared with FEM simulations.
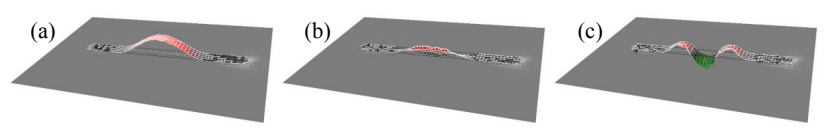

Figure 8: First three measured vibrational modes corresponding to the magnitude peaks ( $a, b$ and $c)$ in Figure 7.

Table 2: Comparison of calculated, simulated, and measured values for the spring constant $(k)$, sensitivity $\left(V_{b} / V_{s} F^{-1}\right)$, and resonance frequency $\left(f_{0}\right)$.

\begin{tabular}{cccc} 
& Calculated & Simulated & Measured \\
\hline$k$ & $18 \mathrm{kN} \mathrm{m}^{-1}$ & $18.7 \mathrm{kN} \mathrm{m}^{-1}$ & N/A \\
$V_{\mathrm{b}} / V_{\mathrm{s}} F^{-1}$ & $1.39 \mathrm{~N}^{-1}$ & $1.34 \mathrm{~N}^{-1}$ & $1.46 \mathrm{~N}^{-1}$ \\
$f_{0}$ & $110 \mathrm{kHz}$ & $103 \mathrm{kHz}$ & $75 \mathrm{kHz}$ \\
\hline
\end{tabular}

\section{CONCLUSION}

A force sensor with a a range of $13 \mathrm{mN}$, a sensitivity of $1.46 \mathrm{~N}^{-1}$, and a bandwidth of $75 \mathrm{kHz}$ has been designed, fabricated, and characterized. Miniaturization and mechanical amplification are essential to achieve these contradicting specifications for the bandwidth and sensitivity. This novel combination allows this sensor to serve in new fields of low force sensing, e.g., the measurement of microscale and microsecond impact behavior of liquids.

The mechanical amplification (and thus sensitivity) can be increased by improving the transmission from sense beam to strain gauge beam (i.e., by cantilever bending). Future work will also focus the development of multiple sensors with different specifications, integration of multiple sensing beams for tactile sensing, and measuring the impact of microdroplets.

\section{ACKNOWLEDGEMENTS}

The authors gratefully acknowledge support by Sectorplan Bèta and Techniek, Europees Fonds voor Regionale Ontwikkeling through the METEORIET project, and the European Research Council (ERC) under the European Union Horizon 2020 Research and Innovation Programme (grant agreement no. 851630).

\section{REFERENCES}

[1] M. Elhadi Ibrahim et al., "Water droplet erosion of wind turbine blades: Mechanics, testing, modeling and future perspectives," Materials, vol. 13, no. 1, p. 157, 2020.

[2] H. Wijshoff, "Drop dynamics in the inkjet printing process," Current Opinion in Colloid \& Interface Science, vol. 36, pp. 20-27, 2018.

[3] S. Mitragotri, "Current status and future prospects of needle-free liquid jet injectors," Nature reviews Drug discovery, vol. 5, no. 7, pp. 543-548, 2006.

[4] A. Mohizin et al., "Impact of the mechanical properties of penetrated media on the injection characteristics of needle-free jet injection," Experimental Thermal and Fluid Science, vol. 126, p. 110396, 2021.

[5] J. C. Stachowiak et al., "Piezoelectric control of needlefree transdermal drug delivery," Journal of Controlled Release, vol. 124, no. 1-2, pp. 88-97, 2007.

[6] A. M. Römgens et al., "Penetration and delivery characteristics of repetitive microjet injection into the skin," Journal of Controlled Release, vol. 234, pp. 98103, 2016.

[7] L. Oyarte Gálvez et al., "Microfluidics control the ballistic energy of thermocavitation liquid jets for needle-free injections," Journal of Applied Physics, vol. 127, no. 10, p. 104901, 2020.

[8] M. R. Prausnitz et al., "Transdermal drug delivery," Nature Biotechnology, vol. 26, no. 11, pp. 1261-1268, 2008.

[9] Y. Zhang et al., "Advances in transdermal insulin delivery," Advanced Drug Delivery Reviews, vol. 139, pp. 51-70, 2019.

[10] K. Cu et al., "Delivery strategies for skin: Comparison of nanoliter jets, needles and topical solutions," Annals of Biomedical Engineering, vol. 48, no. 7, pp. 20282039, 2020.

[11] M. Maroufi et al., "An adjustable-stiffness mems force sensor: Design, characterization, and control," Mechatronics, vol. 56, pp. 198-210, 2018.

[12] Y. Sun et al., "Drosophila flight force measurements using a mems micro force sensor," in The 26th Annual International Conference of the IEEE Engineering in Medicine and Biology Society, vol. 1. IEEE, 2004, pp. 2014-2017.

[13] F. Beyeler et al., "A six-axis mems force-torque sensor with micro-newton and nano-newtonmeter resolution," Journal of Microelectromechanical Systems, vol. 18, no. 2, pp. 433-441, 2009.

[14] W. C. Young et al., Roark's formulas for stress and strain. McGraw-hill New York, 2002, vol. 7.

[15] D. Schubert et al., "Piezoresistive properties of polycrystalline and crystalline silicon films," Sensors and Actuators, vol. 11, no. 2, pp. 145-155, 1987.

[16] M.-H. Bao, Handbook of Sensors and Actuators. Elsevier Science B.V., 2000, vol. 8.

[17] D. Alveringh et al., "A large range multi-axis capacitive force/torque sensor realized in a single soi wafer," in 2014 IEEE 27th International Conference on Micro Electro Mechanical Systems (MEMS). IEEE, 2014, pp. 680-683.

\section{CONTACT}

Dennis Alveringh - d.alveringh@utwente.nl 